BUTP-92/28

COLO-HEP-289

\title{
The Equivalence of the $\mathrm{SU}(N)$ Yang-Mills Theory with a Purely Fermionic Model $\square$
}

\author{
Anna Hasenfratz f \\ University of Arizona Tucson, Department of Physics \\ Tucson, AZ 85721, USA \\ Peter Hasenfratz \\ Institute for Theoretical Physics \\ University of Bern \\ Sidlerstrasse 5, CH-3012 Bern, Switzerland
}

August 2018

\begin{abstract}
We investigate the detailed conditions under which a purely fermionic model with current-current interaction goes over to a renormalizable, asymptotically free $\mathrm{SU}(N)$ gauge theory.
\end{abstract}

\footnotetext{
${ }^{1}$ Work supported in part by Schweizerischer Nationalfonds and NSF Grant PHY-9023257

${ }^{2}$ Present address: Physics Dept., University of Colorado, Boulder CO 80309-390
} 
The idea of composite gauge particles has a long history. The obvious possibility to form composite massless vector particles through vector condensates [1] is plagued by observable Lorentz symmetry breaking [2]. On the other hand, tightly bound vector states, in the limit when their mass goes to zero, are expected to have gauge interactions at low energies. This problem has been investigated by many theorists [2-5] especially in the context of composite $W$ and $Z$ bosons in electroweak interactions [6]. These investigations are phenomenological in the sense that the high energy content of these models is left non-renormalizable, or it is not investigated explicitly further.

It has been demonstrated recently [7-9] that the purely fermionic generalized Nambu-Jona-Lasinio model is completely equivalent to a perturbatively renormalizable quantum field theory which is formulated in terms of elementary scalars and fermions. Although the analytic calculations in [7] refer to the large$N_{\text {colour }}$ limit, the field theoretical basis of the arguments is independent of $N_{c}$. In this letter we discuss the problem of constructing the standard renormalizable $\mathrm{SU}(N)$ Yang-Mills theory out of a purely fermionic model for arbitrary $N \geq 2$. The physical content of the model is the usual gauge invariant selfinteraction of vector gluons. For this reason - rather than talking about bound states and compositeness - one can consider the original fermionic model as a specific realization of the Yang Mills theory. Admittedly, the point of view taken in [7] and here is somewhat different from that of the original idea of composite Higgs [10] or vector bosons [6]. The equivalence of purely fermionic models and theories with elementary scalars and gauge bosons does not have, in itself, phenomenological consequences. We find it intriguing, nevertheless, that the full standard model might be formulated in terms of fermion fields only.

It is easy to understand, how a fermionic theory with 4-fermion interaction might lead to a local gauge theory. Consider the free Lagrangean of $N_{c}$ massive Dirac fermions

$$
\mathcal{L}(x)=\bar{\psi}^{a}(x)\left(\gamma^{\mu} \partial_{\mu}+M\right) \psi^{a}(x) \quad, \quad a=1,2, \ldots, N_{c} .
$$

This model has a $\mathrm{U}\left(N_{c}\right)$ global symmetry with the corresponding conserved currents. Let us demand that the $\mathrm{SU}\left(N_{c}\right)$ Noether currents are pointwise zero, i.e.

$$
j_{\mu}^{A}(x)=\bar{\psi}(x) \gamma^{\mu} T^{A} \psi(x)=0, \forall x, \mu=1, \ldots, d, A=1, \ldots, N_{c}^{2}-1
$$

where $T^{A}$ are the $\mathrm{SU}\left(N_{c}\right)$ generators. The model defined by eqs. (1), (2) has a local $\mathrm{SU}\left(N_{c}\right)$ symmetry. Indeed, the variation of $\mathcal{L}$ under a local $\mathrm{SU}\left(N_{c}\right)$ transformation is proportional to the current $j_{\mu}^{A}(x)$ which is zero according to eq. (2). On the other hand, the constraint $\delta\left(j_{\mu}^{A}(x)\right)$ in the path integral can be represented by a 4 -fermion current-current interaction using $\delta(y) \sim \lim _{\Delta \rightarrow 0} e^{-\frac{1}{\Delta} y^{2}}$. 
In order to investigate further under which circumstances will be the corresponding gauge invariant model a cut-off independent Yang-Mills theory, we shall introduce a regularization and generalize the model slightly. We shall use dimensional regularization and introduce $N_{f}$ fermion fields: $\psi_{i}^{a}(x)$, where $a=1, \ldots, N_{c}$ and $i=1, \ldots, N_{f}$ refer to colour and flavour, respectively. As we discussed before, the Lagrangean

$$
\mathcal{L}=\bar{\psi}\left(\gamma^{\mu} \partial_{\mu}+M\right) \psi+\frac{\kappa^{\epsilon}}{2 m^{2}}\left(\bar{\psi} \gamma^{\mu} T^{A} \psi\right)\left(\bar{\psi} \gamma^{\mu} T^{A} \psi\right)
$$

in the limit $m \rightarrow 0, \epsilon$ fixed, defines a model with local $\mathrm{SU}\left(N_{c}\right)$ symmetry in $n=4-\epsilon$ dimension. Here $\kappa$ is a finite arbitrary mass scale. Introducing the auxiliary fields $W_{\mu}^{A}(x)$ one obtains in the $m \rightarrow 0$ limit

$$
\int D \psi D \bar{\psi} D W_{\mu} \exp \left\{-\int_{x}\left[\bar{\psi}\left(\gamma^{\mu} \partial_{\mu}+M\right) \psi-i \kappa^{\epsilon / 2} W_{\mu}^{A} \bar{\psi} \gamma^{\mu} T^{A} \psi\right] .\right.
$$

The gauge symmetry in eq. (4) is obvious: eq. (4) is the standard, gauge invariant fermion-gluon interaction, but the gluon part $\sim F_{\mu \nu}^{A} F_{\mu \nu}^{A}$ is missing. Integrating over the fermions we get

$$
\int D W_{\mu} \exp \left\{-S\left(W_{\mu}\right)\right\}
$$

where the gauge field action $S\left(W_{\mu}\right)$ is a sum over one-loop graphs with $l$ gluon legs, $l=2,3, \ldots$ :

The first term in eq. (6), giving the quadratic part of the action has the form

$$
\frac{1}{e_{0}^{2}} \int_{p} \frac{1}{2} W_{\mu}^{A}(p) W_{\nu}^{A}(p)\left(p^{2} \delta_{\mu \nu}-p_{\mu} p_{\nu}\right) 6 \int_{0}^{1} d x x(1-x)\left[1+x(1-x) \frac{p^{2}}{M^{2}}\right]^{-\epsilon / 2}
$$

with

$$
e_{0}=\bar{e}_{0} \kappa^{-\epsilon / 2}, \quad \frac{1}{\bar{e}_{0}^{2}}=N_{f} M^{-\epsilon} \frac{2}{3} \frac{1}{(4 \pi)^{n / 2}} \Gamma\left(2-\frac{n}{2}\right) .
$$

For momenta $p^{2} / M^{2} \ll 1$, eq. (7) corresponds to $1 / 4 e_{0}^{2} \int_{x}\left(\partial_{\mu} W_{\nu}^{A}(x)-\partial_{\nu} W_{\mu}^{A}(x)\right)^{2}$. The graphs with 3 and 4 legs in eq. (6) make this expression gauge invariant, the square of the standard field strength tensor will enter. The bare gauge coupling $\bar{e}_{0}^{2}$ is proportional to $\epsilon$, goes to zero as the regularization is removed, as it should in an asymptotically free theory. The one-loop graphs with more than 4 legs in eq. (6) are convergent and are suppressed by $1 / M$ powers for momenta much below $M$. 
In order to proceed further, we have to make a digression and discuss two questions in the standard dimensionally regularized Yang-Mills theory.

A. The relation between the bare coupling and $\Lambda_{\mathrm{MS}}$ Let us consider the action

$$
S^{\mathrm{YM}}=\frac{1}{4} \int F_{\mu \nu}^{A} F_{\mu \nu}^{A} d^{n} x
$$

where

$$
F_{\mu \nu}^{A}=\partial_{\mu} A_{\nu}^{A}-\partial_{\nu} A_{\mu}^{A}-\bar{g}_{0} C^{A B C} A_{\mu}^{B} A_{\nu}^{C},
$$

and $\bar{g}_{0}$ is the bare coupling, $\operatorname{dim} \bar{g}_{0}=(\operatorname{mass})^{-\epsilon / 2}$. The precise way the bare coupling $\bar{g}_{0}$ goes to zero as $\epsilon \rightarrow 0$ will determine the typical scale $(\Lambda$-parameter) of the resulting continuum Yang-Mills theory. Let us tune $\bar{g}_{0}=\bar{g}_{0}(\epsilon)$ towards zero as

$$
\frac{1}{\bar{g}_{0}^{2}}=\left(\frac{2 \beta_{0}}{\epsilon}+\frac{\beta_{1}}{\beta_{0}} \ln \frac{1}{\epsilon}\right) \Lambda^{-\epsilon},
$$

where $\beta_{0}$ and $\beta_{1}$ are the first two universal coefficients of the $\beta$-function and $\Lambda$ is some arbitrary mass. Then the typical low energy scale of the resulting YangMills theory in the $\epsilon \rightarrow 0$ limit will be $\sim \Lambda$. For example, the scale parameter in the MS-scheme is related to $\Lambda$ as

$$
\Lambda_{\mathrm{MS}}=\exp \left(-\frac{\beta_{1}}{2 \beta_{0}^{2}}(1-\ln 2)\right) \Lambda \text {. }
$$

Eq. (12) can be derived as follows. On the two-loop level, eqs. (9), (10) lead to the following relation between the renormalized coupling in the MS-scheme $g_{\mathrm{MS}}(\mu)$ and the bare coupling $\bar{g}_{0}$ :

$$
g_{\mathrm{MS}}^{2}(\mu)=\bar{g}_{0}^{2} \mu^{-\epsilon}+\left(\bar{g}_{0}^{2} \mu^{-\epsilon}\right)^{2} \frac{2 \beta_{0}}{\epsilon}+\left(\bar{g}_{0}^{2} \mu^{-\epsilon}\right)^{3}\left(\frac{\beta_{1}}{\epsilon}+\frac{4 \beta_{0}^{2}}{\epsilon^{2}}\right) .
$$

Eq. (13) contains pole terms only (MS-scheme) and leads to the correct $\beta$ function

$$
\mu \frac{d}{d \mu} g_{\mathrm{MS}}^{2}(\mu)=-\epsilon g_{\mathrm{MS}}^{2}(\mu)-2 \beta_{0} g_{\mathrm{MS}}^{4}(\mu)-2 \beta_{1} g_{\mathrm{MS}}^{6}(\mu)+\ldots
$$

Integrate the renormalization group equation eq. (14) between $\mu$ and $\mu^{\prime}$, where $\mu^{\prime}$ is so large that $g_{\mathrm{MS}}^{2}\left(\mu^{\prime}\right) \ll \epsilon$, and so - according to eq. $(13)-g_{\mathrm{MS}}^{2}\left(\mu^{\prime}\right)=\bar{g}_{0}^{2} \mu^{\prime-\epsilon}$. This way one finds the relation between $\bar{g}_{0}^{2}$ and $g_{\mathrm{MS}}^{2}(\mu)$ which reads

$$
\frac{1}{\bar{g}_{0}^{2} \mu^{-\epsilon}}=\frac{2 \beta_{0}}{\epsilon}+\frac{1}{g_{\mathrm{MS}}^{2}(\mu)}-\frac{\beta_{1}}{\beta_{0}}\left[\ln \left(\frac{\epsilon}{2 \beta_{0} g_{\mathrm{MS}}^{2}(\mu)}\right)+1\right] .
$$

Eqs. (11), (15) and the two-loop definition

$$
\Lambda_{\mathrm{MS}}=\mu\left(\beta_{0} g_{\mathrm{MS}}^{2}(\mu)^{-\beta_{1} / 2 \beta_{0}^{2}} \exp \left\{-\frac{1}{2 \beta_{0} g_{\mathrm{MS}}^{2}(\mu)}\right\}\right.
$$


lead to the relation eq. (12).

B. Large $M$ limit at fixed regularization

Let us add to the action eq. (9) some small gauge invariant perturbation which modifies the quadratic part and the vertices. The perturbation can be local, or nonlocal. We assume that the perturbation depends on some mass scale $M$ in such a way that for any set of fixed momenta flowing into the bare vertices or propagator the perturbation goes to zero as $M \rightarrow \infty$. Consider this modified model and take the limit $M \rightarrow \infty$ at fixed value of the regularization parameter. One expects that the effect of the perturbation disappears in this limit, the Green's functions remain unchanged. Consider any graph before and after the perturbation is introduced and take the difference. For any fixed set of internal and external momenta the integrand of the corresponding momentum integrals goes to zero as $M \rightarrow \infty$. If the regularization is such that the region of momentum integration is constrained (lattice, for example) then the integral itself goes to zero also, and the intuitive expectation is satisfied. The situation is less obvious if dimensional regularization is used and, actually, we are not able to present a formal proof. We shall illustrate on the example of the 1-loop $\beta$ function that the $M \rightarrow \infty$ limit at fixed regularization parameter $\epsilon$ (the precise conditions are given in eqs. (18),(19)) leads to the standard Yang-Mills result, as expected. We shall assume that this is true in every order of perturbation theory.

We return now to the 4-fermion formulation and investigate the structure of the vertices in eq. (5) in more detail. Introduce the rescaled gauge field $A_{\mu}^{A}(x)=1 / e_{0} W_{\mu}^{A}(x)$ and consider a vertex with $l$ external $A$-lines

Here $V_{\mu_{1} \ldots \mu_{l}}^{A_{1} \ldots A_{l}}\left(p_{i}, M\right)=\bar{e}_{0}^{2} N_{f} \cdot f_{\mu_{1} \ldots \mu_{l}}^{A_{1} \ldots A_{l}}\left(p_{i}, M\right)$, where $f$ is a sum of 1-loop integrals

with $l$ massive fermion propagators. Since $\bar{e}_{0}^{2} \sim 1 / N_{f}$ (eq. (8)), $V$ is independent of $N_{f}$. Counting Lorentz indices and dimensions we get that for $p \ll M$ the leading behaviour of $V$ is $\sim M^{4-l}$ for $l=$ even and $\sim p M^{3-l}$ if $l=$ odd. The action in eq. (5) has therefore the following properties: (i) The $l$-point vertex is $\bar{e}_{0}^{l-2} V_{\mu_{1} \ldots \mu_{l}}^{A_{1} \ldots A_{l}}\left(p_{i}, M\right)$, where $V$ is independent of $N_{f}$. (ii) For $p \ll M$, only the 3- and 4-point vertices survive and their form agrees with that of the standard Yang-Mills vertices. (iii) The quadratic part of the action has the standard Yang-Mills form for $p \ll M$. 
Consider now the limit at fixed regularization parameter $\epsilon=4-n$ :

$$
N_{f} \rightarrow \infty, M \rightarrow \infty, N_{f} \cdot M^{-\epsilon}=c(\epsilon), \text { fixed }^{3},
$$

where $c(\epsilon)$ is chosen so that the bare charge in eq. (8) has the form

$$
1 / \bar{e}_{0}^{2}=\left(\frac{2 \beta_{0}}{\epsilon}+\frac{\beta_{1}}{\beta_{0}} \ln \frac{1}{\epsilon}\right) \Lambda^{-\epsilon}
$$

with some arbitrary, fixed scale $\Lambda$. As we discussed in point B, (i)-(iii) and eq. (18) imply that in this limit the standard Yang-Mills model is obtained in $n=4-\epsilon$ dimension with a bare coupling $\bar{e}_{0}^{2}$. Eq. (19) assures then, according to our discussion in point $\mathrm{A}$, that in the $\epsilon \rightarrow 0$ limit a continuum, cut-off independent Yang-Mills theory is obtained whose low energy scale is $\sim \Lambda$. Eqs. (18), (19) give [11]

$$
M=\Lambda \epsilon^{\beta_{1} / 2 \beta_{0}^{2}} \exp \left(\frac{1}{\epsilon} \ln \frac{2 N_{f}}{11 N_{c}}+\frac{1}{2}\left(\Gamma^{\prime}(1)+\ln 4 \pi\right)\right)[1+O(\epsilon)],
$$

showing that for any fixed $\epsilon, M / \Lambda$ goes to infinity as $N_{f} \rightarrow \infty$.

As an illustration, let us discuss the 1-loop $\beta$-function of the model in eq. (5). Since the action $S$ is gauge invariant, the gauge fixing with the FadeevPopov procedure goes through as usual. In Landau gauge, for example, we get for the gluon propagator

$$
D_{\mu \nu}^{A B}(q)=\delta_{A B}\left(\delta_{\mu \nu}-\frac{q_{\mu} q_{\nu}}{q^{2}}\right) \frac{1}{q^{2} \cdot f\left(q^{2}\right)},
$$

where

$$
f\left(q^{2}\right)=6 \int_{0}^{1} d x x(1-x)\left[1+x(1-x) \frac{q^{2}}{M^{2}}\right]^{-\epsilon / 2} .
$$

The ghost propagator and ghost vertex are standard. The 1-loop graphs to the 2- and 3-point functions are also standard (although with the propagator eq. (21) and 3- and 4-point vertices which become non-local at the scale of $M)$, except a single graph in the 3-point function where two legs of the 5point vertex is closed. We define the wave function renormalization and the renormalized coupling $e_{\mathrm{MOM}}(\mu)$ as usual

$$
\begin{gathered}
-\mu^{2} \delta_{A_{1} A_{2}} \delta_{\mu_{1} \mu_{2}}=\left.Z \underset{\mu_{1} \mu_{2}}{Z} \stackrel{(2) A_{1} A_{2}}{\mu_{1}}(p)\right|_{p^{2}=\mu^{2}} \\
i e_{\mathrm{MOM}}(\mu) \mu^{\epsilon / 2} C^{A_{1} A_{2} A_{3}}\left[\delta_{\mu_{1} \mu_{3}}\left(p_{1}-p_{3}\right)_{\mu_{2}}+\delta_{\mu_{1} \mu_{2}}\left(p_{2}-p_{1}\right)_{\mu_{3}}+\delta_{\mu_{2} \mu_{3}}\left(p_{3}-p_{2}\right)_{\mu_{1}}\right]
\end{gathered}
$$

\footnotetext{
${ }^{3}$ i.e. the bare charge is fixed
} 


$$
=\left.Z^{3 / 2} \Gamma \underset{\mu_{1} \mu_{2} \mu_{3}}{(3) / A_{1} A_{2} A_{3}}\left(p_{1}, p_{2}, p_{3}\right)\right|_{\text {s.P. }}
$$

where $\Gamma^{(3) \prime}$ denotes the part of $\Gamma^{(3)}$ which has the same Lorentz structure as the standard Yang-Mills vertex, while the symmetric point (S.P.) is defined by $p_{i}^{2}=\mu^{2}, p_{i} p_{j}=-\frac{1}{2} \mu^{2}, i \neq j$. The renormalized coupling is dimensionless. On dimensional ground we have

$$
e_{\mathrm{MOM}}(\mu) \mu^{\epsilon / 2}=\bar{e}_{0}+\bar{e}_{0}^{3} M^{-\epsilon} f\left(\frac{\mu^{2}}{M^{2}}, \epsilon\right) .
$$

On the left hand side of eq. (25) $\mu$ enters only through the definition (see the left hand side of eq. (24)). On the right hand side of eq. (25) $\mu$ enters through the $\mu$-dependence of the graphs. Since $\mu / M \rightarrow 0$ ( $\epsilon$ fixed $)$, it is clear that the right hand side of eq. (25) can have $\mu$-dependence only through infrared divergencies of the graphs for $\mu \rightarrow 0$. To find these infrared divergencies we can consider small loop momentum. Then, however, we have the standard vertices and propagators (up to $p^{2} / M^{2} \rightarrow 0$ corrections). Consequently, the $\mu$-dependence of eq. (25) is identical to that of the standard Yang-Mills theory:

$$
e_{\mathrm{MOM}}(\mu) \mu^{\epsilon / 2}=\bar{e}_{0}+\bar{e}_{0}^{3}\left[\mu^{-\epsilon}\left(\frac{\beta_{0}}{\epsilon}+a\right)+\mu \text {-independent }\right],
$$

where $a$ is the same finite constant one obtains in the standard Yang-Mills theory. On dimensional ground the $\mu$-independent part in eq. (26) should be proportional to $M^{-\epsilon}$

$$
e_{\mathrm{MOM}}(\mu) \mu^{\epsilon / 2}=\bar{e}_{0}+\bar{e}_{0} \mu^{-\epsilon}\left[\left(\frac{\beta_{0}}{\epsilon}+a\right)+\left(\frac{\mu}{M}\right)^{\epsilon} \cdot b(\epsilon)\right] .
$$

In the limit $M \rightarrow \infty, \epsilon$ fixed (eq. (18)) the last term goes to zero in agreement with the general expectation (see the discussion in point $\mathrm{B}$ above). The relation between $e_{\mathrm{Mом}}(\mu)$ and $\bar{e}_{0}$ will have then the standard form and so will the 1-loop $\beta$-function $\mu \frac{d}{d \mu} e_{\mathrm{MOM}}(\mu)$.

Let us turn back to the form in eq. (3) and discuss an interesting possibility. After a Fierz transformation [12] one can introduce colour singlet auxiliary fields and after integrating over the fermions, $N_{c}$ will enter as an overall factor only. We were not able to make further progress along this line, however.

\section{References}

[1] J.D. Bjorken, Ann. Phys. (N.Y.) 24 (1963) 174. 
[2] T. Banks and A. Zaks, Nucl. Phys. B184 (1981) 303.

[3] M. Veltman, Acta Phys. Pol. B12 (1981) 437;

S. Mandelstam, A Passion in Physics, (C. DeTar et al. eds.) World Scientific, Singapore 1985, p.97.

[4] I. Bialynicki-Birula, Phys. Rev. 130 (1963) 465;

G.S. Guralnik, Phys. Rev. 136 (1964) 1404;

T. Eguchi and H. Sugawara, Phys. Rev. D10 (1974) 4257;

T. Eguchi, Phys. Rev. D14 (1976) 2755;

C. Bender, F. Cooper and G. Guralnik, Ann. Phys. (N.Y.) 109 (1977) 165.

[5] M. Suzuki, Phys. Rev. D37 (1988) 210; A. Cohen, H. Georgi and E. H. Simmons, Phys. Rev. D38 (1988) 405.

[6] M. Peskin, in Proc. 1981 Int. Symp. of Lepton and Photon Interactions (Bonn, 1981, W. Pfeil ed.);

B. Schrempp, in Proc. of XXIII Int. Conf. on High Energy Physics, Berkeley, 1986 (S.C. Loken, ed.) World Scientific, Singapore 1987, p. 454.

[7] A. Hasenfratz, P. Hasenfratz, K. Jansen, J. Kuti and Yue Shen, Nucl. Phys. B365 (1991) 79.

[8] J. Zinn-Justin, Saclay, preprint SPhT/91/092.

[9] J. Soto, Phys. Lett. B280 (1992) 75.

[10] V.A. Miransky, T. Tanabashi and K. Yamawaki, Mod. Phys. Lett A4 (1989) 1043;

W.A. Bardeen, C.T. Hill and M. Lindner, Phys. Rev. D41 (1990) 1647.

[11] This and some other results of this paper have been reported (without a proper discussion of the limit in eq. (18), however) in

P. Hasenfratz, Proc. of the Int. Workshop on Electroweak Symmetry Breaking, Hiroshima, 1991.

[12] Fierz transformation in the context of dimensional regularization has been discussed by

M. Blatter, Bern preprint BUTP-92/25. 